\title{
Mixed Carcinoid-Mucinous Adenocarcinoma Arising in Mature Teratoma of Mesentery
}

\author{
Su-Jin Shin · Eun-Mi Son · Chang Ohk Sung · Kyu-Rae Kim \\ Department of Pathology, Asan Medical Center, University of Ulsan College of Medicine, Seoul, Korea
}

Malignant transformation of mature cystic teratoma is uncommon, and occurs in approximately $2 \%-4 \%$ of cases. ${ }^{1}$ The most common tumor is squamous cell carcinoma, followed by mucinous carcinoma, carcinoid tumor, thyroid carcinoma, etc.; however, any of the tissues in mature teratoma may undergo malignant transformation. ${ }^{1}$

Goblet cell carcinoid (mucinous carcinoid) is a distinctive neoplasm with features of both carcinoid tumor and adenocarcinoma. Most cases described in the literature have occurred in the appendix and rarely, in the ovary. Although mature cystic teratomas occur in many extragonadal areas including the mesentery $^{2}$ and greater omentum, occurrence of goblet cell carcinoid arising in extragonadal teratoma has been rarely described, with only a single case in mediastinum being reported in the English literature. ${ }^{3}$ Moreover, a combination of mucinous adenocarcinoma, goblet cell carcinoid, carcinoid tumor, and mature teratoma in an extragenital organ has not been reported.

Herein, we present a rare case of combined mucinous adenocarcinoma and goblet cell and typical carcinoid tumor arising in mature cystic teratoma of the mesentery in a 48-year-old woman.

\section{CASE REPORT}

A 48-year-old woman presented with a palpable abdominal mass in the periumbilical area with a vague abdominal pain of

\section{Corresponding Author}

Kyu-Rae Kim, M.D.

Department of Pathology, Asan Medical Center, University of Ulsan College of

Medicine, 88 Olympic-ro 43-gil, Songpa-gu, Seoul 138-736, Korea

Tel: +82-2-3010-4514, Fax: +82-2-472-7898, E-mail: krkim@amc.seoul.kr

Received: June 30, 2014 Revised: September 3, 2014

Accepted: September 17, 2014 one month duration. Past medical history was unremarkable. She had no symptoms of carcinoid syndrome. On physical examination, the abdomen was markedly distended due to a solid movable mass. Abdominal and pelvic computed tomography scans revealed a huge heterogeneously enhanced mass in the abdominal cavity, which was associated with multiple smaller nodules throughout the entire abdominal and pelvic cavities and the right lobe of the liver. Bilateral ovaries were diffusely enlarged to form a semisolid mass. Laboratory findings demonstrated increased serum levels of carcinoembryonic antigen (264 $\mathrm{ng} / \mathrm{mL}$; normal $<6 \mathrm{ng} / \mathrm{mL}$ ) and carbohydrate antigen 125 (294 $\mathrm{U} / \mathrm{mL}$, normal $<35 \mathrm{U} / \mathrm{ml}$ ). Alpha-fetoprotein, beta-subunit of human chorionic gonadotropin and carcinohydrate antigen-19-9 were within normal range.

Exploratory laparotomy revealed a huge mass in the mesentery of the small intestine (Fig. 1A), measuring $20 \times 18 \times 11 \mathrm{~cm}$, with coexisting multiple outbulging hepatic masses, bilateral ovarian masses, and diffusely seeding nodules on the peritoneal surface and omentum. On cut section, the mesenteric mass had solid and cystic portions. The cystic portion, occupying approximately $60 \%$ of the tumor, was filled with yellow-tan sebumlike materials, hair, mucoid and gelatinous substances, while the solid portion ( $40 \%$ ) was composed of loose myxoid tissue (Fig. 1B). Bilateral ovaries were enlarged, measuring $12.5 \times 8 \times$ $6 \mathrm{~cm}$ in the right ovary and $10 \times 6 \times 3 \mathrm{~cm}$ in the left ovary, and multiple gelatinous tumor nodules were observed on both ovarian surfaces and parenchyma (Fig. 1C). The cut surface of the ovarian masses was greyish white and gelatinous. The seeding nodules of the peritoneal surface and omentum had myxoid and gelatinous appearances. Excision of the mesenteric mass, bilateral salpingo-oophorectomy, total omentectomy and appendectomy were undertaken. Intraoperative frozen sections were per- 

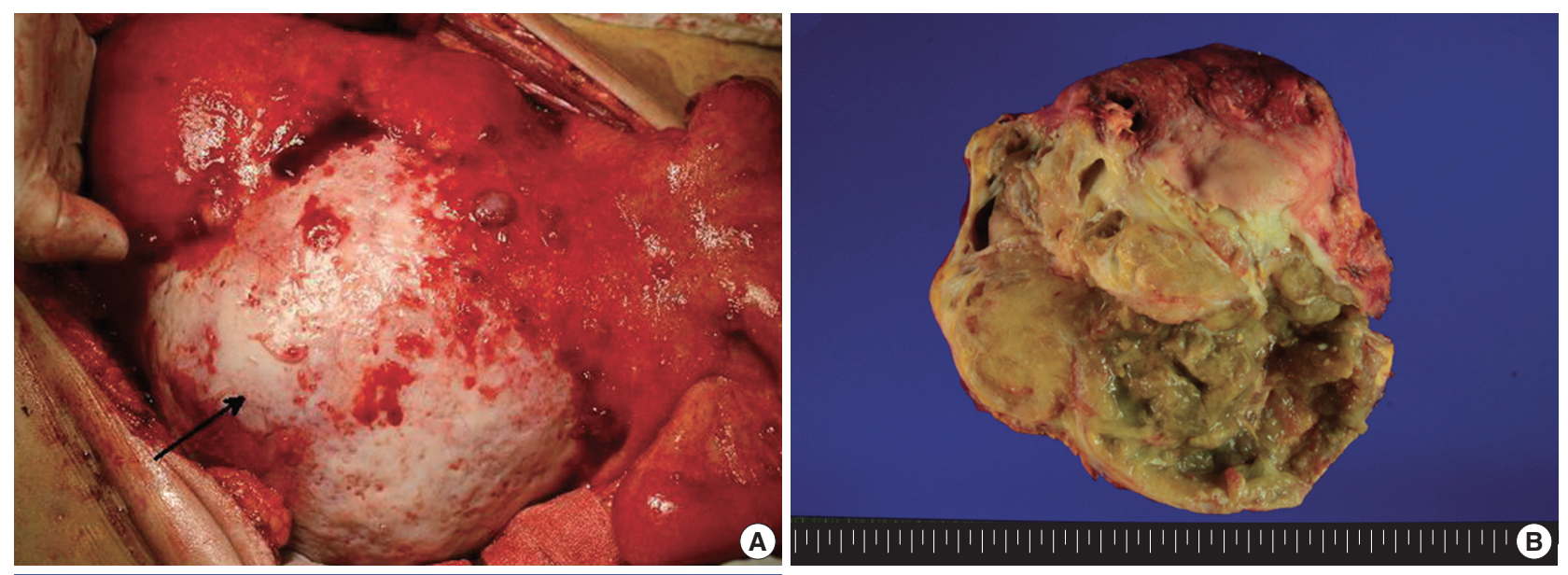

Fig. 1. External appearance (A) and cut surface (B) of the mesenteric teratoma showing solid and cystic portions with yellow sebum-like materials in the cystic space of the mass. Arrow indicates mesenteric mass. Partially disrupted ovarian mass (C) showing multiple gelatinous tumor nodules on the surface and parenchyma (l, tumor implant; S, ovarian surface).

formed for the ovarian mass, and the diagnosis was mucinous adenocarcinoma.

On microscopic examinations, the mesenteric tumor was encapsulated by thick fibrous tissue. The cystic portion of the mesenteric mass showed characteristic histologic features of mature teratoma containing various mature tissues derived from all three germ layers, including skin and its appendages, salivary glands, cartilage, adipose tissue, and smooth muscle bundles (Fig. 2A). The adjacent solid portion showed various histologic features of carcinoid tumors; a 1.5-cm sized well-circumscribed nodule showing insular, small glandular, rosettoid, and solid tubular patterns similar to those of midgut (insular) carcinoid tumors, thin cord-like or anastomosing trabecular patterns resembling hindgut (trabecular) carcinoid tumors, and dissecting mucin pools containing floating tumor cells (Fig. 2A, B), suggesting goblet cell carcinoid tumor. The insular and trabecular carcinoid tumor cells were composed of uniformly small and round nuclei with finely stippled chromatin, and abundant cytoplasm with eosinophilic granules. Mitotic figures were rare in insular and trabecular carcinoid areas (Ki-67 labeling index was less

than 2\%). The tumor cells within mucin pools were individually scattered or formed small nests having signet ring cell-like or goblet cell appearances, abundant foamy cytoplasm, low nuclear cytoplasmic ratio, and flattened nuclei pushed to the periphery (Fig. 2B). There was mild variation in nuclear sizes, mitotic figures were rarely identified, and $\mathrm{Ki}-67$ labeling index was low (less than 2\%) (Fig. 2B). On immunohistochemical stains, the trabecular and insular carcinoid tumor components showed strong and diffuse immunoreactivity for synaptophysin (Fig. 2C) and focal immunoreactivity for chromogranin. The tumor cells within mucin pools showed multifocal immunopositivity for synaptophysin (Fig. 2B inset).

In the vicinity of the carcinoid tumors, there were mucin pools containing signet ring cell-like or goblet cells showing significantly enlarged nuclei with high nuclear cytoplasmic ratio, marked nuclear pleomorphism, prominent nucleoli, and frequent mitotic figures (Fig. 2D). Immunohistochemical stain for synaptophysin was completely lost in the malignant signet ring celllike tumor cells, and proliferating cells defined by Ki-67 immunohistochemical stains were significantly increased, up to $20 \%$, 


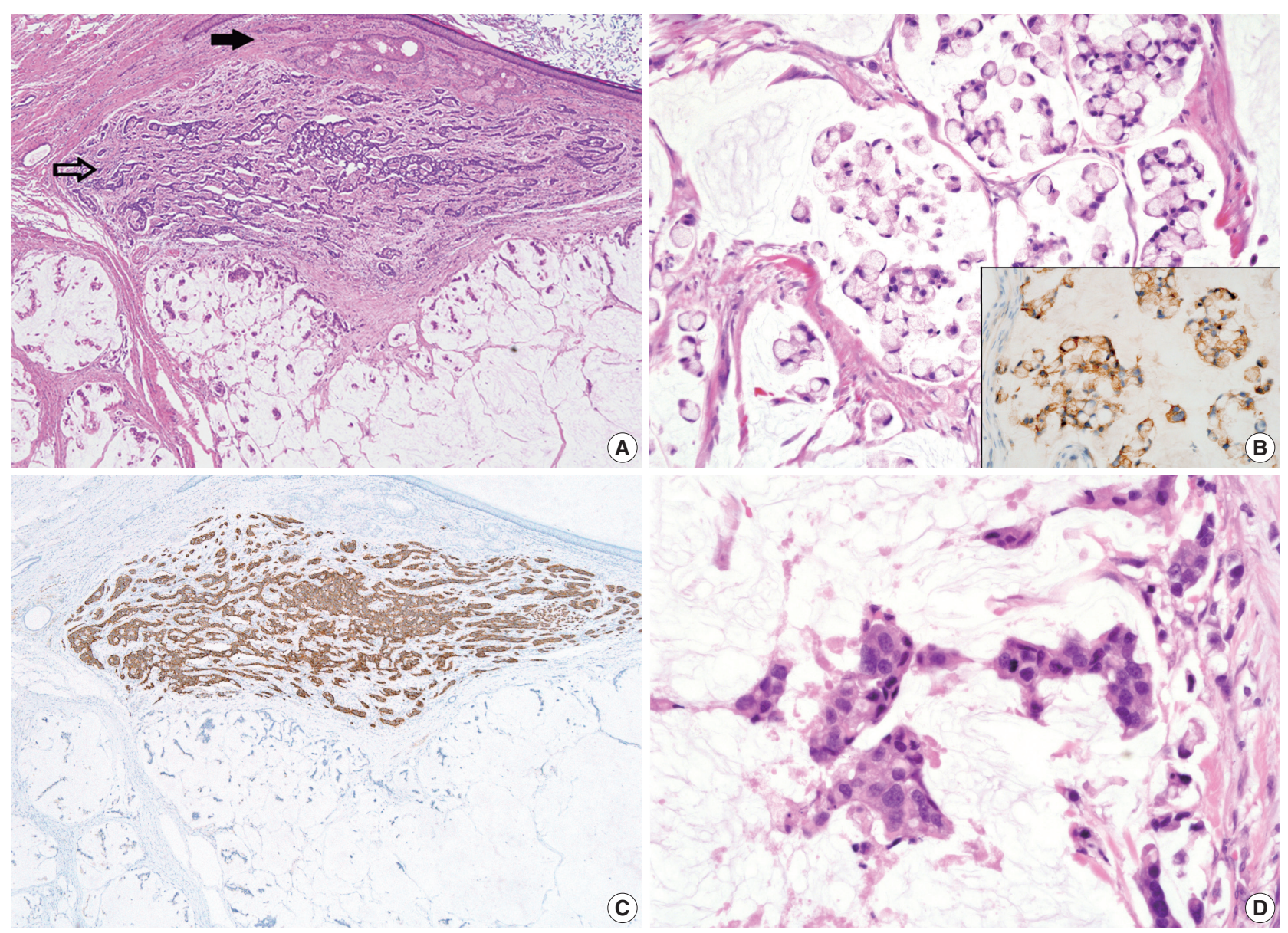

Fig. 2. Histopathologic findings of mesenteric mass showing mature cystic teratoma containing a mature teratomatous component (A, closed arrow), an area of trabecular carcinoid (A, open arrow), goblet cell carcinoid (B) showing immunopositivities for synaptophysin (B inset and C), and mucinous adenocarcinoma with signet ring cells within dissecting mucin pools (D). Note a high nuclear cytoplasmic ratio and significant nuclear pleomorphism in the mucinous adenocarcinoma component (D) in contrast to low nuclear cytoplasmic ratio and flattened nuclei in goblet cell carcinoid (B).

in those malignant cells. The two areas containing the signet ring cell-like appearance with and without nuclear pleomorphism were intermingled with each other and the border could not be clearly delineated.

Both ovaries were mostly replaced by tumor tissue with a multinodular appearance. Each nodule was well-circumscribed and was composed of a mucin pool containing signet ring cells identical to those in the mesenteric mass (Fig. 3A). Extraovarian mucin pools were also noted in the omentum and serosal surfaces of the pelvic organs, forming pseudomyxoma peritonei (Fig. 3B). There were no insular or trabecular carcinoid areas in either ovarian tumor.

The patient received five cycles of adjuvant chemotherapy with paclitaxel and cisplatin, but she died of multiorgan metastasis eight months postoperatively.

\section{DISCUSSION}

Historically, a tumor describing mixed glandular and neuroendocrine neoplasm has had many synonyms, including goblet cell carcinoid, mucinous carcinoid, adenocarcinoid, microglandular carcinoma, intermediate cell carcinoid, amphicrine neoplasia, and composite tumor. There remains confusion and debate regarding the terminologies amongst pathologists and clinicians. In the appendix and ovaries, intimately admixed glandular and neuroendocrine neoplasm composed of goblet cells, neuroendocrine cells, or hybrid cells containing both mucin and neuroendocrine granules floating in pools of mucin are designated as goblet cell carcinoid. Goblet cell carcinoid tumors in the appendix present in four types: 1) pure and uniform tubules composed entirely of goblet cells; 2) goblet cell carcinoid with 

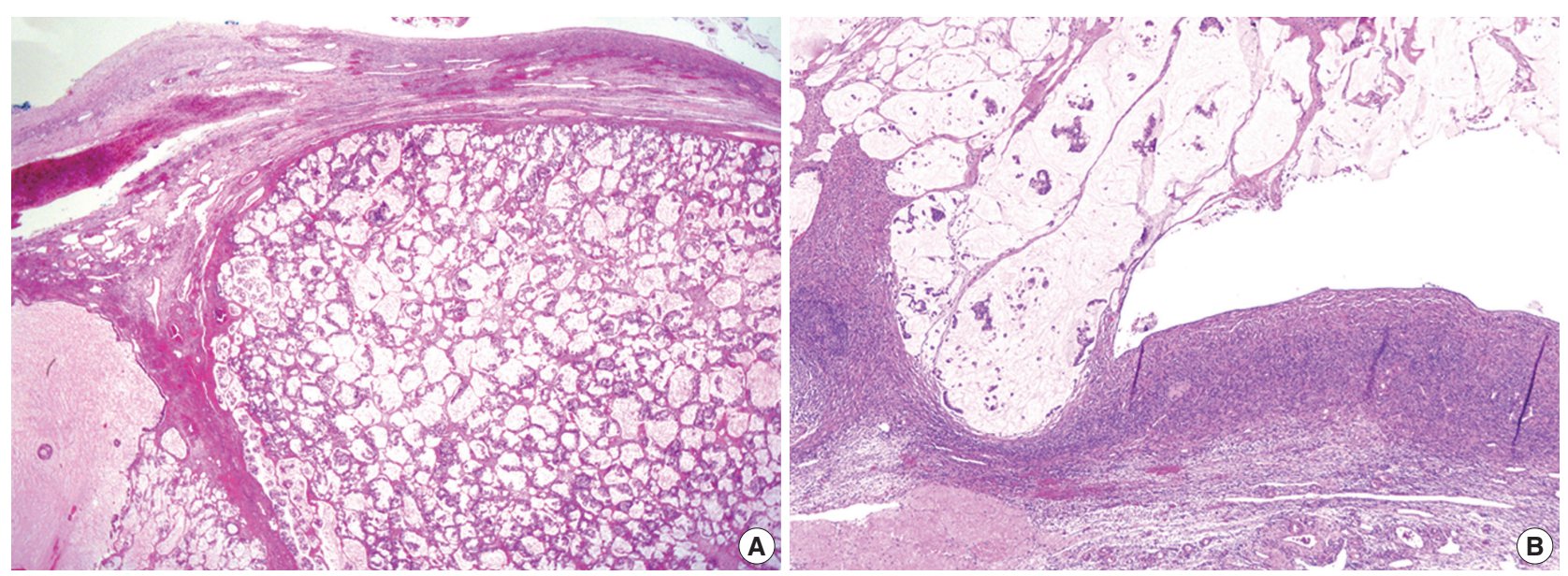

Fig. 3. Histopathologic findings of ovarian mass. The tumor tissue shows a multinodular appearance with well-circumscribed margins. Each nodule is composed of a mucin pool containing malignant tumor cells identical to those in the mesenteric mass (A). The tumor tissue in the ovarian surface and superficial cortex formed pseudomyxoma peritonei composed of mucin pools with floating signet ring cells, which are connected to the extraovarian mucin pools in the pelvic cavity (B).

adenocarcinomatous area (adenocarcinoma ex goblet cell carcinoid); 3) goblet cell carcinoid with mucinous cystadenoma; and 4) the combined classical carcinoid with goblet cell carcinoid tumor. ${ }^{4}$ In our case, goblet cell carcinoid was combined with mucinous adenocarcinoma and additional typical insular and trabecular carcinoid tumor. Histologically, it is not easy to distinguish the pure goblet cell carcinoid from mucinous adenocarcinoma arising in goblet cell carcinoid tumors because of the same goblet cell morphology in mucin pools in both tumors and intimately admixed tumor components in most cases. Goblet cell carcinoid tumors have more aggressive clinical behaviors, showing metastasis in $20 \%$ of cases, ${ }^{5}$ compared to the classic type of carcinoid tumor, but the tumors have relatively favorable prognosis compared to the mucinous adenocarcinoma ex goblet cell carcinoid. ${ }^{6}$ Pools of mucin are usually small in goblet cell carcinoid and they usually lie within the glands or in the periglandular stroma. The nuclear atypia is not significant in goblet cell carcinoid; however, mucinous adenocarcinoma ex goblet cell carcinoid has greater nuclear pleomorphism, higher mitotic rate, and Ki-67 proliferating activity, which can lead to a diagnosis of mucinous adenocarcinoma ex goblet cell carcinoid.

Histogenetically, two neoplasms in adjacent but separate areas are designated as a collision tumor of a biclonal derivation, while composite tumors having intimate admixture of the two components are believed to result from bidirectional differentiation of a single neoplasm. A molecular study suggested that goblet cell carcinoid has similar expression patterns of genes for secretory and Paneth cell differentiation to those of mucinous cystadenomas and colonic adenocarcinomas; thus, goblet cell carcinoids are closer to colonic adenocarcinomas than to classical carcinoids, ${ }^{7}$ implicating that the goblet cell carcinoid probably arises in progenitor cells of the epithelial crypt.

Rarely, mature cystic teratomas have been described in the mesentery and greater omentum. ${ }^{2}$ The pathogenetic origin is explained as primitive germ cells entrapped during embryonic development, a supernumerary (ectopic) ovary located in the omentum, ${ }^{8}$ or secondary implantation of a teratomatous component to the omentum following the torsion and rupture of ovarian teratoma. ${ }^{9}$

The diagnosis of extra-appendiceal and extraovarian goblet cell carcinoid should be determined after thorough examination of the two organs and exclusion of appendiceal or ovarian origins. The patient in our case received an appendectomy at the same time, which was thoroughly examined, but only serosal tumor implants were identified. Mucinous adenocarcinoma involved bilateral ovaries in our case; however, the ovaries were thought to be metastatic sites because of their bilateral multinodular appearance, and involvement of the ovarian surface and superficial cortex of the ovary only. A significant amount of mucin pools dissecting the ovarian stroma and signet ring cell-like morphology are extremely rare in primary mucinous adenocarcinoma of the ovary. ${ }^{10}$ Moreover, the existence of mesenteric mature teratoma and significantly larger extraovarian mucin pools forming pseudomyxoma peritonei are supporting features of extraovarian origin.

This case had combined features of several rare findings: primary mesenteric mature teratoma associated with carcinoid tu- 
mor, coexistence of all three types of carcinoid tumors including insular, trabecular and goblet cell carcinoid, overgrown mucinous adenocarcinoma of goblet cell morphology in the background of goblet cell carcinoid, metastasis of extragonadal goblet cell carcinoid to the ovaries, and formation of pseudomyxoma peritonei from extra-appendiceal neoplasm, but the clinical course followed those of mucinous adenocarcinoma and high grade pseudomyxoma peritonei. Recognition of mucinous adenocarcinoma components seems to be closely correlated with the patient's prognosis. Thus, careful histological observation is required to make a clinically relevant diagnosis.

\section{Conflicts of Interest}

No potential conflict of interest relevant to this article was reported.

\section{REFERENCES}

1. Kurman RJ, Ellenson LH, Ronnett BM. Blaustein's pathology of the female genital tract. 6th ed. New York: Springer, 2011; 877.

2. Papakonstantinou E, Iavazzo C, Hasiakos D, Kleanthis CK, Fotiou S, Kondi-Pafiti A. Extraovarian mature cystic teratoma of the mesentery: a case report and literature review. Clin Exp Obstet Gynecol 2011; 38: 291-3.
3. Lancaster KJ, Liang CY, Myers JC, McCabe KM. Goblet cell carcinoid arising in a mature teratoma of the mediastinum. Am J Surg Pathol 1997; 21: 109-13.

4. Chetty R, Klimstra DS, Henson DE, Albores-Saavedra J. Combined classical carcinoid and goblet cell carcinoid tumor: a new morphologic variant of carcinoid tumor of the appendix. Am J Surg Pathol 2010; 34: 1163-7.

5. Toumpanakis C, Standish RA, Baishnab E, Winslet MC, Caplin ME. Goblet cell carcinoid tumors (adenocarcinoid) of the appendix. Dis Colon Rectum 2007; 50: 315-22.

6. Turaga KK, Pappas SG, Gamblin T. Importance of histologic subtype in the staging of appendiceal tumors. Ann Surg Oncol 2012; 19: 1379-85.

7. van Eeden S, Offerhaus GJ, Hart AA, et al. Goblet cell carcinoid of the appendix: a specific type of carcinoma. Histopathology 2007; 51: 763-73.

8. Abrego D, Ibrahim AA. Mesenteric supernumerary ovary. Obstet Gynecol 1975; 45: 352-3.

9. Kearney MS. Synchronous benign teratomas of the greater omentum and ovary: case report. Br J Obstet Gynaecol 1983; 90: 676-9.

10. Lee KR, Young RH. The distinction between primary and metastatic mucinous carcinomas of the ovary: gross and histologic findings in 50 cases. Am J Surg Pathol 2003; 27: 281-92. 